\title{
Quantum Hall effective action for the anisotropic Dirac semimetal
}

\author{
Carlos Hoyos $\odot,{ }^{1}$ Ruben Lier, ${ }^{2}$ Francisco Peña-Benitez $\odot,{ }^{2}$ and Piotr Surówka ${ }^{2}$ \\ ${ }^{1}$ Department of Physics and Instituto de Ciencias y Tecnologías Espaciales de Asturias (ICTEA), Universidad de Oviedo, \\ c/Federico García Lorca 18, ES-33007 Oviedo, Spain \\ ${ }^{2}$ Max-Planck-Institut für Physik komplexer Systeme and Würzburg-Dresden Cluster of Excellence ct.qmat, \\ Nöthnitzer Str. 38, 01187 Dresden, Germany
}

(Received 8 July 2020; revised 6 August 2020; accepted 12 August 2020; published 28 August 2020)

\begin{abstract}
We present a study of Hall transport in semi-Dirac critical phases. The construction is based on a covariant formulation of relativistic systems with spatial anisotropy. Geometric data together with external electromagnetic fields is used to devise an expansion procedure that leads to a low-energy effective action consistent with the discrete $\mathcal{P} \mathcal{T}$ symmetry that we impose. We use the action to discuss terms contributing to the Hall transport and extract the coefficients. We also discuss the associated scaling symmetry.
\end{abstract}

DOI: 10.1103/PhysRevB.102.081303

\section{INTRODUCTION}

Low-energy effective actions have become an important tool to study topological responses of quantum phases of matter [1]. The predictive power of effective actions comes from the fact that they mainly rely on the symmetries of the system in question, hiding our ignorance about the microscopic details of the system in a set of parameters. Although initially applied to phases with large symmetry groups such as Galilean or Poincaré, in recent years effective theories have been used to shed light on states with more exotic or reduced symmetry groups. These symmetries can be realized in various topological materials undergoing quantum phase transitions from a conductor to a band insulator. They emerge when topological defects occurring at isolated points in momentum space collide. Such defects are symmetry protected points where valence and conduction bands touch.

An interesting class corresponds to the quantum critical point connecting a band insulator and a graphenelike state ${ }^{1}$ (see Fig. 1). The critical phase, known as the semi-Dirac phase, is semimetallic with electrons dispersing linearly in one direction and quadratically in the other [2-10]. Examples of systems that exhibit such phases in two dimensions include $\mathrm{TiO}_{2} / \mathrm{VO}_{2}$ heterostructures [11], (BEDT-TTF) ${ }_{2} I_{3}$ organic salts under pressure [12], photonic metamaterials [13], and certain non-Hermitian systems [14]. Semi-Dirac phases reveal distinct features, for example, in transport phenomena $[15,16]$ or driven by light [17-19].

\footnotetext{
${ }^{1}$ At the critical point, the $\pm \pi$ Berry's phase of each Dirac cone annihilate.

Published by the American Physical Society under the terms of the Creative Commons Attribution 4.0 International license. Further distribution of this work must maintain attribution to the author(s) and the published article's title, journal citation, and DOI. Open access publication funded by the Max Planck Society.
}

In [5] it has been shown numerically that when a magnetic field is applied to these systems, the topological response of such phases is governed by two independent nondissipative viscosities, in contrast to isotropic phases where a single Hall viscosity is present $[20,21]$. However, the method did not provide an explanation for the vanishing of a possible third viscosity, which is allowed by the continuous symmetries.

To shed light on the generic low-energy properties of such quantum Hall states, we construct an effective action for systems with a semi-Dirac phase. Our construction contributes to the quest of understanding Hall viscosities; nondissipative transport coefficients that emerge in the context of topological order [21-27], fluid dynamics [28-40], or active matter [41-46]. Initially thought of as an elusive transport property, Hall viscosity has been experimentally identified in both hard [47] and soft [48] condensed matter experiments.

In this Rapid Communication we present a step-by-step construction of the low-energy effective theory. First we study the coupling of fermions to the background geometry and gauge fields in a microscopic model. The coupling to the curved background is not unique, unless extra geometric constraints are invoked [49], but this ambiguity is hidden in the coefficients of the effective action. In the following section we present the main construction of the effective action including symmetries, derivative counting, and contributing terms up to the second order in the expansion. The main properties of the effective action derive from the breaking of the would-be $2+1$ Lorentz group of a graphenelike phase to a $1+1$ Lorentz subgroup by a spatial vector that marks the anisotropic direction, and by an unbroken discrete $\mathcal{P} \mathcal{T}$ symmetry. The technical details are relegated to the Supplemental Material [50]. Finally, we present the topological transport of the semi-Dirac states that follows from our effective action. We show that a hitherto overlooked transport coefficient, due to antisymmetric strains, is necessarily present in such phases. We close with a discussion of the results. 


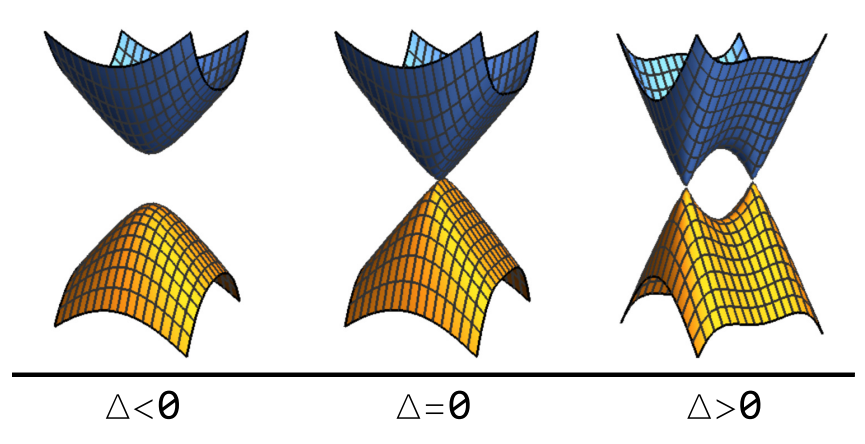

FIG. 1. Spectrum of the Hamiltonian. $\Delta=0$ corresponds to the semi-Dirac critical point.

\section{THE SEMI-DIRAC SYSTEM}

The low-energy Hamiltonian describing the anisotropic semi-Dirac semimetal reads

$$
\mathcal{H}=\mathbf{d}(\mathbf{p}) \cdot \boldsymbol{\sigma}
$$

where $\boldsymbol{\sigma}$ is a vector of Pauli matrices. $\mathbf{d}(\mathbf{p})=\left(p_{x}, 0, \frac{p_{y}^{2}}{2 m}-\right.$ $\Delta$ ) with $m$ being a parameter with dimensions of mass. In Fig. 1 we show the three phases captured by the Hamiltonian equation (2.1), where the semi-Dirac phase is obtained when $\Delta=0$.

When we study the coupling to background fields it is more convenient to use the action formalism

$$
S=\int d^{3} x\left[i \bar{\psi} \gamma^{a} \mathcal{P}_{a}^{b} \partial_{b} \psi-\bar{\psi} M\left(\Delta, l^{a} \partial_{a}\right) \psi\right],
$$

where $\gamma^{a}=\left(\sigma_{3},-i \sigma_{2}, i \sigma_{1}\right)$ are $2+1$ Dirac matrices. One can view the system as having an effective Dirac mass which is momentum dependent, i.e., $M\left(\Delta, l^{a} \partial_{a}\right)=\Delta-\frac{1}{2 m}\left(l^{a} \partial_{a}\right)^{2}$. The form of the action naturally implies that transverse derivatives must be of the same order as longitudinal derivatives squared. We aim to have the theory in a covariant form, which will facilitate putting the electrons on a curved space-time. Therefore we have introduced the anisotropy vector $l^{a}=$ $(0,0,1)$, and the transverse projector

$$
\mathcal{P}_{a}^{b}=\delta_{a}^{b}-l_{a} l^{b}, \quad l^{a} l_{a}=1 .
$$

$l^{a}$ breaks the $S O(2,1)$ Lorentz symmetry present for standard relativistic fermions down to $S O(1,1) \times C_{2}$, where $S O(1,1)$ corresponds to boosts in the plane transverse to $l^{a}$ and $C_{2} 180^{\circ}$ rotations on the spacelike plane containing vector $l^{a}$. In fact, such a discrete rotation is equivalent to the transformation $l^{a} \rightarrow-l^{a}$. The discrete symmetries can be understood from the point of view of a graphenelike model as proposed in [5]. Ignoring the spin microscopic degrees of freedom, it consists of two species of fermions organized by valleys on two hexagonal sublattices. Such fermions exhibit parity $\mathcal{P}$ and time-reversal symmetry $\mathcal{T}$. Anisotropy stems from the mass deformation that breaks separate symmetries, however, preserving the $\mathcal{P} \mathcal{T}$ combination. In addition there is the graphene's inversion symmetry that we refer to as $P^{*} \simeq C_{2}$ that acts by sending $\vec{x} \rightarrow-\vec{x}$, and simultaneously interchanging the sublattice atoms and valleys [51].
In the semi-Dirac phase $\Delta=0$ the action enjoys an additional anisotropic scaling symmetry

$$
\mathcal{P}_{a}^{b} x_{b} \rightarrow \lambda^{2} \mathcal{P}_{a}^{b} x_{b}, \quad l^{a} x_{a} \rightarrow \lambda l^{a} x_{a}, \quad \psi \rightarrow \lambda^{-3 / 2} \psi .
$$

The scaling dimensions deduced from the fermionic action for the derivatives and gauge potentials are

$$
\left[\mathcal{P}_{a}^{b} \partial_{b}\right]=\left[\mathcal{P}_{a}^{b} A_{b}\right]=2, \quad\left[l^{a} \partial_{a}\right]=\left[l^{a} A_{a}\right]=1 .
$$

Then, for $l^{a}=(0,0,1)$ the field strengths scaling dimensions are $\left[E_{y}\right]=[B]=3$ and $\left[E_{x}\right]=4$, while the energy has dimensions $[E]=\left[\partial_{t}\right]=2$. The scale invariance is reflected on the dependence of the energy of Landau levels with the magnetic field $E \sim B^{2 / 3}[2,52]$. The derivative expansion we propose below will contain terms $\partial_{t} / \omega_{c} \sim \ell_{B}^{x} \partial_{x} \sim O\left(\epsilon^{2}\right)$ and $\ell_{B}^{y} \partial_{y} \sim$ $O(\epsilon)$, for $\epsilon \sim\left(B / m^{2}\right)^{-1 / 3} \ll 1$. The cyclotron frequency and magnetic lengths are defined (up to constant factors) as

$$
\omega_{c}=\frac{1}{\ell_{B}^{x}}=\left(\frac{B^{2}}{m}\right)^{1 / 3} \sim \frac{m}{\epsilon^{2}}, \quad \ell_{B}^{y}=\frac{1}{(m B)^{1 / 3}} \sim \frac{\epsilon}{m} .
$$

\section{COUPLING TO BACKGROUND FIELDS}

As already pointed out, our goal is to construct the generating functional of the semi-Dirac system in the presence of a magnetic field. To do so, it is necessary to couple the fermionic action equation (2.2) to external $U(1)$ gauge fields and a curved background which will allow us to predict the response of the $n$-point functions of the $U(1)$ and the stress energy tensor by functional differentiation.

First we will introduce a background metric $g_{\mu \nu}$ and an orthonormal basis $e_{a}$ of tangent vectors, $e_{a}{ }^{\mu} g_{\mu \nu} e_{b}{ }^{\nu}=\eta_{a b}$ (inverse vielbeins). In addition we have introduced a set of dual cotangent one forms $E^{a}$ (vielbeins), such that

$$
\left\langle E^{a}, e_{b}\right\rangle=\delta_{b}^{a} .
$$

The metric can be determined by the vielbeins $g_{\mu \nu}=$ $\eta_{a b} E_{\mu}^{a} E_{\nu}^{b}$. Then, the generalization of Eq. (2.2) to curved space-time involves replacing the derivative, Dirac matrices, and vector $l^{a}$ in flat space by their pullbacks to the tangent space $\partial_{a}=e_{a}{ }^{\mu} \partial_{\mu}$, etc.

Actually, given the anisotropic nature of the system it is convenient to split the vielbeins into their transverse and longitudinal parts:

$$
\begin{gathered}
\hat{e}_{a}{ }^{\mu}=\mathcal{P}_{a}^{b} e_{b}{ }^{\mu}, \quad \hat{E}^{a}{ }_{\mu}=\mathcal{P}_{b}^{a} E^{b}{ }_{\mu}, \\
l^{\mu}=l^{a} e_{a}{ }^{\mu}, \quad l_{\mu}=l_{a} E^{a}{ }_{\mu} .
\end{gathered}
$$

It is also convenient to decompose the $2+1$ Poincare generators $\left(P_{a}, J_{a b}\right)$ in terms of the broken and unbroken ones, therefore we introduce the following splitting:

$$
\begin{gathered}
\hat{K}=\frac{1}{2} \hat{\epsilon}^{a b} J_{a b}, \quad \hat{\jmath}_{a}=J_{a b} l^{b}, \\
\pi=P_{a} l^{a}, \quad \hat{p}_{a}=P_{b} \mathcal{P}_{a}^{b},
\end{gathered}
$$

where the transverse fully antisymmetric tensor is defined as $\hat{\epsilon}^{a b}=\epsilon^{a b c} l_{c}{ }^{2} . \pi, p_{a}$ generate longitudinal and transverse

\footnotetext{
${ }^{2}$ We define $\epsilon^{a b c}$ as the fully antisymmetric symbol, therefore $\varepsilon^{\mu \nu \rho}=$ $\epsilon^{a b c} e_{a}{ }^{\mu} e_{b}{ }^{v} e_{c}{ }^{\rho}$ has components $\sqrt{-g} \varepsilon^{012}=1$.
} 
translations, respectively, and $\hat{K}$ is the $S O(1,1)$ boost generator. The broken generators have been collected in $\hat{J}_{a}$.

The standard way of minimally coupling relativistic fermions to gauge fields and curved space-time relies on replacing the partial derivative by a covariant one:

$$
\partial_{\mu} \rightarrow \mathcal{D}_{\mu} \equiv \partial_{\mu}+i e A_{\mu}+\frac{1}{2} \omega^{a b}{ }_{\mu} J_{a b},
$$

where $A_{\mu}$ is the $U(1)$ gauge field, $J^{a b}=\frac{1}{4}\left[\gamma^{a}, \gamma^{b}\right]$ the Lorentz algebra generators, and $\omega^{a b}{ }_{\mu}$ the so-called Levi-Civita spin connection, defined as

$$
\omega^{a b}{ }_{\mu}=-E^{a}{ }_{\nu} \nabla_{\mu} e^{b \nu},
$$

where we set the torsion to be vanishing and choose $\nabla_{\mu}$ to be a covariant derivative constructed with the Christoffel symbols. Notice that this definition is covariant under the full $S O(2,1)$ group of local Lorentz transformations, while the system is only invariant under the $S O(1,1)$ subgroup that keeps the vector $l^{a}$ fixed, so this is not the most general possible form of the covariant derivative. To generalize it, it is convenient to split the spin connection into its longitudinal and transverse parts,

$$
\omega^{a b}{ }_{\mu}=\hat{\omega}_{\mu} \hat{\epsilon}^{a b}+2 \hat{\theta}^{[a}{ }_{\mu} l^{b]},
$$

where $\hat{\omega}_{\mu}=\frac{1}{2} \hat{\epsilon}_{a b} \omega^{a b}{ }_{\mu}$ and $\hat{\theta}^{a}{ }_{\mu}=\omega^{a b}{ }_{\mu} l_{b}$, which after plugging it into the covariant derivative produces

$$
\mathcal{D}_{\mu}=\partial_{\mu}+i e A_{\mu}+\hat{\omega}_{\mu} \hat{K}+\hat{\theta}^{a}{ }_{\mu} \hat{J}_{a} .
$$

In particular, $\hat{\theta}^{a}{ }_{\mu}$ takes the form

$$
\hat{\theta}^{a}{ }_{\mu}=\hat{e}^{a v} T_{\mu \nu}+l_{\mu} \hat{e}^{a v} T_{\nu},
$$

where $T_{\mu \nu}=\partial_{\mu} l_{\nu}-\partial_{\nu} l_{\mu}$ and $T_{\mu}=l^{\nu} T_{\nu \mu}$. Actually, when a Lorentz transformation generated by $\hat{K}$ is applied,

$$
\hat{E}_{\mu}^{a} \rightarrow \Omega(x) \hat{\epsilon}_{b}^{a} \hat{E}_{\mu}^{b}, \quad l_{\mu} \rightarrow l_{\mu},
$$

the transverse part of the spin connection transforms as an Abelian connection

$$
\hat{\omega}_{\mu} \rightarrow \hat{\omega}_{\mu}+\partial_{\mu} \Omega
$$

whereas $\hat{\theta}^{a}{ }_{\mu}$ transforms as a vector.

At this point we are ready to couple the fermionic field to the background geometry, introducing a more general covariant derivative which transforms properly under $S O(1,1)$ boost and local $U(1)$ gauge transformations,

$$
\mathcal{D}_{\mu}=\partial_{\mu}-i A_{\mu}+\frac{1}{2} \omega_{\mu}{ }^{a b} J_{a b}+\alpha \hat{\theta}_{\mu}{ }^{a} \hat{\jmath}_{a},
$$

where $\alpha$ is an unknown coupling constant, whose value could be determined by finding an UV completion. However, from our effective field theory perspective, its specific value is irrelevant, because it would just be hidden in the particular values of the coefficients appearing in the effective action. With this covariant derivative we can write the gauge and diffeomorphism invariant action

$$
S=\int \sqrt{-g}\left[i \bar{\psi} \gamma^{a} \hat{e}_{a}{ }^{\mu} \mathcal{D}_{\mu} \psi-\Delta \bar{\psi} \psi+\frac{1}{2 m} l^{\mu} l^{\nu} \mathcal{D}_{\mu} \bar{\psi} \mathcal{D}_{\nu} \psi\right]
$$

The action is invariant under local gauge, diffeomorphism, and boost transformations, that act on the external fields as follows:

$$
\begin{gathered}
\delta A_{\mu}=\mathcal{L}_{\chi} A_{\mu}+\partial_{\mu} \Lambda, \\
\delta l_{\mu}=\mathcal{L}_{\chi} l_{\mu}, \\
\delta \hat{e}_{a}{ }^{\mu}=\mathcal{L}_{\chi} \hat{e}_{a}{ }^{\mu}+\Omega \hat{\epsilon}_{a}{ }^{b} \hat{e}_{b}{ }^{\mu},
\end{gathered}
$$

where $\mathcal{L}_{\chi}$ is the Lie derivative along the vector $\chi^{\mu}=\xi^{a} \hat{e}_{a}{ }^{\mu}+$ $\kappa l^{\mu}$. Given the symmetry transformations it is possible to construct the covariant tensors $F_{\mu \nu}=2 \partial_{[\mu} A_{\nu]}, \hat{R}_{\mu \nu}=2 \partial_{[\mu} \hat{\omega}_{\nu]}$, $T_{\mu \nu}=2 \partial_{[\mu} l_{\nu]}$, which will be used as the building blocks of the effective field theory we will discuss below. We will collectively refer to them as $X_{\mu \nu}$. In addition, we also have the Riemann tensor built from the metric, $R^{\mu}{ }_{v \rho \lambda}$.

In fact, we would like to emphasize that $l_{\mu}$ can be interpreted as the source that couples to the momentum current along the anisotropic direction. Therefore, the two form $T_{\mu \nu}$ corresponds to its field strength, in analogy with the electromagnetic field $\left(F_{\mu \nu}\right)$ and the gauge field. Alternatively, $T_{\mu \nu}$ appears in the proposal of [49] as the torsion on a NewtonCartan-like space-time.

\section{THE EFFECTIVE ACTION}

In a quantum Hall state the fermions are gapped by the magnetic field and can be integrated out. The resulting effective action will be a local functional of the background sources, that must be invariant under the local symmetry transformations (3.15) and discrete symmetries of the fermionic action, in particular $\mathcal{P} \mathcal{T}$. Local invariance can be made manifest using covariant terms to construct the effective action. Discrete symmetries are then used to determine which of these terms are allowed. Once these are identified, the strategy will be to organize them in a derivative expansion, assuming the fields are slowly varying with respect to the characteristic length and time scales of the problem. Given this, one can write down all possible terms up to a required order in the expansion and include the combinations that respect the discrete symmetries. We present the technical details in the Supplemental Material [50].

The derivative expansion is organized according to the physics of Landau levels in the semi-Dirac semimetal. Consistent with the anisotropic scaling of the fermionic action, we assign different orders to transverse and longitudinal derivatives $\mathcal{P}_{a}^{b} \partial_{b} \sim O\left(\epsilon^{2}\right), l^{a} \partial_{a} \sim O(\epsilon)$, where $\epsilon \ll 1$ is a parameter we use to characterize the order. In general, the terms we present can have contributions at different orders in the expansion, so the order we assign always refers to the contribution of lowest possible order.

The magnetic field determines the gap, therefore we assign $B \sim O\left(\epsilon^{0}\right)$. Moreover, we expect the chemical potential to be finite and nonzero, $A_{t} \sim O\left(\epsilon^{0}\right)$. Finally, in analogy with the isotropic case, we assume that the electric field is small compared to the magnetic field, $E_{x} \sim E_{y} \sim O(\epsilon)$. This implies the following order in the expansion for the vector potentials $A_{x} \sim B y \sim O\left(\epsilon^{-1}\right), A_{y} \sim O\left(\epsilon^{0}\right)$. The metric and vielbeins are always nonvanishing, even when the geometry is flat, so $E^{a} \sim e_{a} \sim O\left(\epsilon^{0}\right)$. This implies that the components of 
the transverse spin connection will be higher order than the electric fields $\hat{\omega}_{\mu} \sim O\left(\epsilon^{2}\right)$.

Now we use the fact that there is a vector $l_{\mu}$ (with transverse projector $\mathcal{P}_{\mu}{ }^{\nu}$ ).

For each field strength $X_{\mu \nu}(X=F, \hat{R}, T)$ we can define two vectors and a scalar:

$$
X_{\mu}=l^{\alpha} X_{\alpha \mu}, \quad \tilde{X}_{\mu}=\mathcal{P}_{\mu \alpha} \varepsilon^{\alpha \nu \lambda} X_{\nu \lambda}, \quad X_{\perp}=\varepsilon^{\mu \nu \lambda} l_{\mu} X_{\nu \lambda} .
$$

Note that the norm of $F_{\mu}$ (or the dual version $\tilde{F}_{\mu}$ ) is $\sim B$, so the order in the derivative expansion is $O\left(\epsilon^{0}\right)$. With the norm we can define an $O\left(\epsilon^{0}\right)$ scalar $\mathcal{B}_{y}^{2}=F_{\mu} F^{\mu} \sim l_{y}^{2} B^{2}$. Moreover, one can construct a second $O\left(\epsilon^{0}\right)$ scalar using $F_{\perp}: \mathcal{B}_{0}^{2}=F_{\perp}^{2} \sim$ $l_{0}^{2} B^{2}$. We will refer to them collectively as $\mathcal{B}_{A}$.

Armed with the above formalism we are in a position to write down the effective action. To the lowest order $O\left(\epsilon^{0}\right)$ there are just two possible terms in the action

$$
\mathcal{L}_{0}=-\mathcal{E}\left(\mathcal{B}_{A}\right)+\frac{v}{4 \pi} \varepsilon^{\mu \nu \lambda} A_{\mu} \partial_{\nu} A_{\lambda} .
$$

The first term is an arbitrary function of $\mathcal{B}_{A}$. This can be thought of as energy density of the quantum Hall state. The second term is the topological Chern-Simons term. At the next level in the expansion $[O(\epsilon)]$ the terms that respect $\mathcal{P} \mathcal{T}$ symmetry are

$$
\mathcal{L}_{1}=\sum_{i=1}^{6} c_{i}^{\mathcal{P} \mathcal{T}}\left(\mathcal{B}_{A}\right) S_{i}^{\mathcal{P} \mathcal{T}}
$$

where

$$
\begin{aligned}
& S^{\mathcal{P} \mathcal{T}}=\left\{S_{1}, S_{2}, F_{\perp} S_{3}, F_{\perp} S_{4}, S_{5}, S_{6}\right\}, \\
& c^{\mathcal{P} \mathcal{T}}\left(\mathcal{B}_{A}\right)=\left\{c_{1}, c_{2}, \bar{c}_{3}, \bar{c}_{4}, c_{5}, c_{6}\right\},
\end{aligned}
$$

and we define

$$
\begin{aligned}
& S_{1}=l^{\mu} \partial_{\mu} \mathcal{B}_{y}, \quad S_{2}=l^{\mu} \partial_{\mu} \mathcal{B}_{0}, \quad S_{3}=F^{\mu} T_{\mu}, \quad S_{4}=\tilde{F}^{\mu} T_{\mu}, \\
& S_{5}=\varepsilon^{\mu \nu \lambda} F_{\mu} \partial_{\nu} F_{\lambda}, \quad S_{6}=\varepsilon^{\mu \nu \lambda} F_{\mu} \partial_{\nu} \tilde{F}_{\lambda} .
\end{aligned}
$$

The anisotropy leads to a plethora of new terms at $O\left(\epsilon^{2}\right)$, which we list in [50]. However, given our interest in topological transport we note that out of all these terms the one associated with the so-called Euler current $\sim A_{\mu} J_{E}^{\mu}(u)$ is of particular importance because it gives rise to topological transport [53,54]. The current is defined using the Riemann tensor and unit norm vectors $u^{\mu}, u^{\mu} u_{\mu}=\sigma$, where $\sigma= \pm 1$,

$$
J_{E}^{\mu}(u)=\frac{1}{8 \pi} \varepsilon^{\mu \nu \lambda} \varepsilon^{\alpha \beta \gamma} u_{\alpha}\left(\nabla_{\nu} u_{\beta} \nabla_{\lambda} u_{\gamma}+\frac{\sigma}{2} R_{\nu \lambda \beta \gamma}\right) .
$$

From all the possible terms there are only three independent ones corresponding to $u^{\mu} \propto l^{\mu}, F^{\mu}, \widetilde{F}^{\mu}$, and only the last one contributes to $\mathcal{P} \mathcal{T}$ preserving topological transport with a coefficient $\kappa$. Another $O\left(\epsilon^{2}\right)$ term that will be relevant is $T_{\perp}$, which is of the form of a torsional Hall viscosity involving only the longitudinal projection of the vielbeins. ${ }^{3}$ An analogous term was discussed in [49].

\footnotetext{
${ }^{3}$ Even though the torsion field $T^{a}{ }_{\mu \nu}=-2\left(\partial_{[\mu} E_{\nu]}^{a}-\omega_{b[v}^{a} E_{\mu]}^{b}\right)$ vanishes by construction, the anisotropy allows one to introduce the Lorentz invariant one form $l_{\mu}=l_{a} E^{a}{ }_{\mu}$ with field strength $T_{\mu \nu}$.
}

Having an effective action we can now proceed to study the responses of an anisotropic quantum Hall state to external perturbations.

\section{HALL TRANSPORT}

We apply the formalism developed so far to study DC responses to electric field, strain, and vorticity. Among these responses are topological responses whose importance stems from the fact that they often provide us with universal characteristics of quantum states. Such universal features are indispensable for a better understanding of strongly coupled fractional Hall states.

We define the transverse and longitudinal components of the stress tensor from the variation of the effective action with respect to vielbeins and gauge fields:

$$
\delta S=-\int d^{3} x \sqrt{-g}\left(\tau_{\mu}{ }^{a} \delta e_{a}{ }^{\mu}+j^{\mu} \delta A_{\mu}\right) .
$$

The simplest of these responses is the Hall conductivity. It follows from the Chern-Simons term and it is given by

$$
\sigma_{x y}=\frac{v}{2 \pi},
$$

which is the same as in the isotropic case. We will next focus on the response of the stress tensor to applied strains. In our geometric formulation it will correspond to the metric fluctuations. Such fluctuations can be easily embedded in our construction as independent variations of vielbeins. A second variation of the action with respect to vielbeins gives the stress-stress two-point functions from which the transport coefficients can be extracted via Kubo formulas for the viscosity tensor through appropriate projections [55]

$$
\eta_{a b}^{\mu v}=\lim _{\omega \rightarrow 0} \frac{i}{\omega}\left\langle\tau_{a}^{\mu} \tau_{b}^{v}\right\rangle
$$

Below we show that anisotropic states preserving $\mathcal{P} \mathcal{T}$ symmetry can have two independent Hall contributions to the viscosity tensor corresponding to such responses. The vielbein variations naturally include nonisotropic and nonsymmetric viscous responses. Therefore our formulation is analogous to related frameworks that introduce a nonzero torsion [49,5661]. Before extracting the coefficients we note that the timereversal odd viscosity tensor in a $\mathcal{P} \mathcal{T}$-invariant theory can be decomposed as follows:

$$
\eta^{i j k l}=8 \eta^{\mathrm{iso}} P_{\mathrm{iso}}^{i j k l}+8 \eta^{\mathrm{nem}} P_{\mathrm{nem}}^{i j k l}+8 \eta^{\mathrm{vor}} P_{\mathrm{vor}}^{i j k l},
$$

where the projection tensors ${ }^{4}$ are defined as

$$
\begin{aligned}
& P_{\mathrm{iso}}^{i j k l}=-\frac{1}{16}\left[\epsilon^{i k} \delta^{j l}+(i \leftrightarrow j)+(k \leftrightarrow l)+(i k \leftrightarrow j l)\right], \\
& P_{\mathrm{nem}}^{i j k l}=\frac{1}{8} l^{\left(i \tilde{l}^{j}\right)} \delta^{l k}-(i j \leftrightarrow k l), \\
& P_{\mathrm{vor}}^{i j k l}=\frac{1}{8} l^{\left[{ }^{[} \tilde{l}^{j]}\right.} \delta^{l k}-(i j \leftrightarrow k l),
\end{aligned}
$$

\footnotetext{
${ }^{4}$ The tensors are orthogonal to each other and the parity-even part of the viscosity tensor with respect to the all-index contraction; however, they do not satisfy the $P^{2}=P$ condition, usually implied by the name projector.
} 
where $\tilde{l}^{i}=\epsilon^{i j} l_{j}$. Using the above formulas we can extract Hall responses in the semi-Dirac phase [50]. The first coefficient is a modification of the isotropic contribution

$$
\eta^{\text {iso }}=\frac{\kappa}{4 \pi} B-\frac{1}{2} c_{5} B^{2}-\frac{1}{2} f_{11} .
$$

In addition there is another Hall viscosity encoding responses of symmetric strains, present due to the anisotropy. These properties are characteristic for the nematic phase so we refer to it as nematic [62].

$$
\eta^{\mathrm{nem}}=\frac{1}{2} c_{5} B^{2}+\frac{1}{2} f_{11}
$$

We do not find any other anisotropic contribution to the symmetric part of the viscosity tensor in the semi-Dirac phase, although arguments based on a continuous symmetry group do not forbid a third coefficient. Finally we identify a hitherto neglected response to vorticity that appears in the semi-Dirac phase:

$$
\eta^{\mathrm{vor}}=\frac{1}{2} c_{5} B^{2}-\frac{1}{2} f_{11}
$$

however, notice that this term is also responsible for a response in the antisymmetric stress tensor due to symmetric strain.

In general, the coefficients are arbitrary functions of the $B$ field and $\Delta$. For $\Delta \ll B$ the dependence will be fixed by the scaling symmetry of the semi-Dirac point, up to corrections suppressed by $\Delta / B$. The Chern-Simons and Euler current terms have dimensionless coefficients, while

$$
c_{5} \sim B^{-1}, \quad f_{11} \sim B .
$$

Therefore, at the semi-Dirac point both odd viscosities have a similar dependence with the magnetic field $\eta_{T} \sim \eta_{I} \sim B$, but deviations are expected for $\Delta \neq 0$.

\section{DISCUSSION}

Recent interest in odd responses is stimulated by experiments with fluids that break parity. Such fluids emerge in the context of electron hydrodynamics as well as biological setups that can be modeled by fluid membranes. Anisotropy can naturally appear for such flows and could modify flow solutions and physical outcome.
Motivated by quantum Hall physics of semi-Dirac critical phases we have developed a formalism that allows one to write down effective actions for such systems. A key ingredient in the construction is a covariant prescription allowing a coupling to geometry. The anisotropy is generated by a spatial vector that we treat as an independent field. Equipped with this construction we proposed an expansion scheme that allowed us to write down an effective action for the semiDirac phase. As an application of the formalism we calculated odd responses for this system showing independent contributions to the viscosity tensor. We noted that $\mathcal{P} \mathcal{T}$ symmetry restricts the components to two independent coefficients. Finally, we showed that semi-Dirac materials are natural hosts for torsional-like responses.

The couplings in the effective action can depend on the microscopic physics of the system, in particular on the band topology. As long as the topological bands do not spoil the semi-Dirac symmetries, our construction will be robust. In general all the coefficients in the effective action (except the filling fraction and $\kappa$ ) can vary continuously with the ratio $\left|\Delta / B^{2 / 3}\right|$, as long as both quantities are nonzero. For $\left|\Delta / B^{2 / 3}\right| \ll 1$ (in units of $m$ ) we expect a universal dependence of the coefficients with the magnetic field, with powers that are fixed by the scaling symmetry of the semi-Dirac phase. In the opposite limit one abandons the regime of the semi-Dirac phase, so the derivative expansion we have introduced is not expected to remain valid.

Semi-Dirac phases can also be found in $3+1$ dimensions. In principle, this method can be used to systematically construct the effective theory for such systems as well. This program has already been initiated to describe anomaly-induced transport $[49,63]$.

\section{ACKNOWLEDGMENTS}

We thank C. Copetti and K. Landsteiner for discussions. R.L., F.P.-B., and P.S. acknowledge financial support by the Deutsche Forschungsgemeinschaft (DFG, German Research Foundation) under Germany's Excellence Strategy through Würzburg-Dresden Cluster of Excellence on Complexity and Topology in Quantum Matter-ct.qmat (EXC 2147, project ID 390858490). F.P.-B. acknowledges the Quantum Matter Academy of ct.qmat for support. C.H. has been partially supported by the Spanish Grant No. PGC2018-096894-B100 and by the Principado de Asturias through Grant No. GRUPIN-IDI/2018/000174.
[1] T. H. Hansson and T. K. Kvorning, in Strongly Coupled Field Theories for Condensed Matter and Quantum Information Theory, Springer Proceedings in Physics Vol. 239 (Springer International Publishing, Cham, 2020), pp. $1-68$.

[2] S. Banerjee, R. R. P. Singh, V. Pardo, and W. E. Pickett, Phys. Rev. Lett. 103, 016402 (2009).

[3] P. Delplace and G. Montambaux, Phys. Rev. B 82, 035438 (2010)

[4] P. Dietl, F. Piéchon, and G. Montambaux, Phys. Rev. Lett. 100, 236405 (2008).
[5] F. Peña-Benitez, K. Saha, and P. Surówka, Phys. Rev. B 99, 045141 (2019).

[6] M. D. Uryszek, E. Christou, A. Jaefari, F. Krüger, and B. Uchoa, Phys. Rev. B 100, 155101 (2019).

[7] L. Tarruell, D. Greif, T. Uehlinger, G. Jotzu, and T. Esslinger, Nature (London) 483, 302 (2012).

[8] S. A. Sato, J. W. McIver, M. Nuske, P. Tang, G. Jotzu, B. Schulte, H. Hübener, U. De Giovannini, L. Mathey, M. A. Sentef, A. Cavalleri, and A. Rubio, Phys. Rev. B 99, 214302 (2019).

[9] B. Roy and M. S. Foster, Phys. Rev. X 8, 011049 (2018). 
[10] A. Mawrie and B. Muralidharan, Phys. Rev. B 99, 075415 (2019).

[11] V. Pardo and W. E. Pickett, Phys. Rev. Lett. 102, 166803 (2009).

[12] S. Katayama, A. Kobayashi, and Y. Suzumura, J. Phys. Soc. Jpn. 75, 054705 (2006).

[13] Y. Wu, Opt. Express 22, 1906 (2014).

[14] A. Banerjee and A. Narayan, arXiv:2001.11188.

[15] J. M. Link, B. N. Narozhny, E. I. Kiselev, and J. Schmalian, Phys. Rev. Lett. 120, 196801 (2018).

[16] I. Mandal and K. Saha, Phys. Rev. B 101, 045101 (2020).

[17] A. Narayan, Phys. Rev. B 91, 205445 (2015).

[18] K. Saha, Phys. Rev. B 94, 081103(R) (2016).

[19] K. Kitayama and M. Mochizuki, Phys. Rev. Research 2, 023229 (2020).

[20] J. E. Avron, J. Stat. Phys. 92, 543 (1998).

[21] J. E. Avron, R. Seiler, and P. G. Zograf, Phys. Rev. Lett. 75, 697 (1995).

[22] N. Read, Phys. Rev. B 79, 045308 (2009).

[23] C. Hoyos, Int. J. Mod. Phys. B 28, 1430007 (2014).

[24] C. Hoyos and D. T. Son, Phys. Rev. Lett. 108, 066805 (2012).

[25] O. Golan, C. Hoyos, and S. Moroz, Phys. Rev. B 100, 104512 (2019).

[26] F. Rose, O. Golan, and S. Moroz, SciPost Phys. 9, 006 (2020).

[27] J. Böttcher, C. Tutschku, L. W. Molenkamp, and E. M. Hankiewicz, Phys. Rev. Lett. 123, 226602 (2019).

[28] P. S. Alekseev, Phys. Rev. Lett. 117, 166601 (2016).

[29] L. V. Delacrétaz and A. Gromov, Phys. Rev. Lett. 119, 226602 (2017); 120, 079901(E) (2018).

[30] A. Lucas and P. Surówka, Phys. Rev. E 90, 063005 (2014).

[31] E. Kogan, Phys. Rev. E 94, 043111 (2016).

[32] S. Ganeshan and A. G. Abanov, Phys. Rev. Fluids 2, 094101 (2017).

[33] I. Matthaiakakis, D. Rodríguez Fernández, C. Tutschku, E. M. Hankiewicz, J. Erdmenger, and R. Meyer, Phys. Rev. B 101, 045423 (2020).

[34] B. N. Narozhny and M. Schütt, Phys. Rev. B 100, 035125 (2019).

[35] T. Holder, R. Queiroz, and A. Stern, Phys. Rev. Lett. 123, 106801 (2019).

[36] S. Pu, M. Fremling, and J. K. Jain, Phys. Rev. Research 2 , 013139 (2020).

[37] P. Rao and B. Bradlyn, Phys. Rev. X 10, 021005 (2020).

[38] P. Wiegmann and A. G. Abanov, Phys. Rev. Lett. 113, 034501 (2014).

[39] M. F. Lapa and T. L. Hughes, Phys. Rev. E 89, 043019 (2014).
[40] A. G. Abanov and G. M. Monteiro, Phys. Rev. Lett. 122, 154501 (2019).

[41] D. Banerjee, A. Souslov, A. G. Abanov, and V. Vitelli, Nat. Commun. 8, 1573 (2017).

[42] G. Salbreux and F. Jülicher, Phys. Rev. E 96, 032404 (2017).

[43] A. Souslov, A. Gromov, and V. Vitelli, Phys. Rev. E 101, 052606 (2020).

[44] D. Banerjee, A. Souslov, and V. Vitelli, arXiv:2005.00621.

[45] D. Banerjee, V. Vitelli, F. Jülicher, and P. Surówka, arXiv:2002.12564.

[46] T. Markovich and T. C. Lubensky, arXiv:2006.05662.

[47] A. I. Berdyugin, S. G. Xu, F. M. D. Pellegrino, R. K. Kumar, A. Principi, I. Torre, M. B. Shalom, T. Taniguchi, K. Watanabe, I. V. Grigorieva, M. Polini, A. K. Geim, and D. A. Bandurin, Science 364, 162 (2019).

[48] V. Soni, E. S. Bililign, S. Magkiriadou, S. Sacanna, D. Bartolo, M. J. Shelley, and W. T. M. Irvine, Nat. Phys. 15, 1188 (2019).

[49] C. Copetti and K. Landsteiner, Phys. Rev. B 99, 195146 (2019).

[50] See Supplemental Material at http://link.aps.org/supplemental/ 10.1103/PhysRevB.102.081303 for technical details related with discrete symmetries, the derivative expansion, and computation of the viscosity tensor from the effective action.

[51] V. Gusynin, S. Sharapov, and J. Carbotte, Int. J. Mod. Phys. B 21, 4611 (2007).

[52] K. Esaki, M. Sato, M. Kohmoto, and B. I. Halperin, Phys. Rev. B 80, 125405 (2009).

[53] S. Golkar, M. M. Roberts, and D. T. Son, J. High Energy Phys. 12 (2014) 138.

[54] S. Golkar, M. M. Roberts, and D. T. Son, J. High Energy Phys. 04 (2015) 110.

[55] B. Bradlyn, M. Goldstein, and N. Read, Phys. Rev. B 86, 245309 (2012).

[56] T. L. Hughes, R. G. Leigh, and E. Fradkin, Phys. Rev. Lett. 107, 075502 (2011).

[57] T. L. Hughes, R. G. Leigh, and O. Parrikar, Phys. Rev. D 88, 025040 (2013).

[58] M. Valle, J. High Energy Phys. 07 (2015) 006.

[59] K. Jensen, SciPost Phys. 5, 011 (2018).

[60] C. Tutschku, J. Böttcher, R. Meyer, and E. Hankiewicz, Phys. Rev. Research 2, 033193 (2020).

[61] J. de Boer, J. Hartong, E. Have, N. A. Obers, and W. Sybesma, SciPost Phys. 9, 018 (2020).

[62] Y. You, G. Y. Cho, and E. Fradkin, Phys. Rev. X 4, 041050 (2014).

[63] C. Copetti, J. High Energy Phys. 01 (2020) 190. 